\title{
Monascin accelerates anoikis in circulating tumor cells and prevents breast cancer metastasis
}

\author{
KUNG-YEN CHEN ${ }^{1}$, JUI-AN LIN ${ }^{1,2}$, HAN-YUN YAO ${ }^{1}$, AN-CHIH HSU ${ }^{1}$, YU-TING TAI ${ }^{1,2}$ and BING-YING HO ${ }^{3}$ \\ ${ }^{1}$ Department of Anesthesiology, Wan Fang Hospital, Taipei Medical University, Taipei 11696; \\ ${ }^{2}$ Department of Anesthesiology, School of Medicine, College of Medicine, Taipei Medical University, Taipei 11031; \\ ${ }^{3}$ Department of General Surgery, Wan Fang Hospital, Taipei Medical University, Taipei 11696, Taiwan
}

Received April 26, 2019; Accepted July 28, 2020

DOI: $10.3892 / 01.2020 .12029$

\begin{abstract}
Anoikis resistance has been observed in various types of cancers in which anchorage-independent growth is a crucial step for cancer metastasis. Therefore, agents interfering with this specific cancer cell behavior may be integrated into novel antimetastatic strategies. Monascin (MS), a secondary metabolite found in Monascus species, is a known potent chemopreventive compound used for treating metabolic complications; however, the effect of MS on anoikis resistance has not been investigated. In this study, 4T1 breast cells were treated with MS under either suspension or adhesion conditions. The higher cytotoxicity of MS was more potent against suspended cells than against adherent cells. This selective cytotoxicity was due to the induction of anoikis, which was evidenced by changes in cell aggregation, caspase activity, and Annexin V/propidium iodide binding as well as the results of systemic metastasis in an animal model. Furthermore, MS inhibited E-cadherin and $\beta$-catenin expression in the cells; the treated cells formed spherical aggregates, which suggested that anchorage-independent growth was prevented by MS. These results provide new insights into the mechanisms underlying the growth-preventing effect of MS on cancer cells and indicate the potential ability of MS to suppress metastasis.
\end{abstract}

\section{Introduction}

Surgery is the treatment of choice for patients with breast cancer. However, the presence of circulating tumor cells (CTCs) after surgery has been reported to be significantly associated with early recurrence (1). The release of tumor cells into

Correspondence to: Dr Bing-Ying Ho, Department of General Surgery, Wan Fang Hospital, Taipei Medical University, 111 Xinglong Road, Section 3, Taipei 11696, Taiwan

E-mail: 105113@w.tmu.edu.tw

Dr Yu-Ting Tai, Department of Anesthesiology, Wan Fang Hospital, Taipei Medical University, 111 Xinglong Road, Section 3, Taipei 11696, Taiwan

E-mail: yttai@w.tmu.edu.tw

Key words: monascin, anoikis, circulating tumor cell, metastasis peripheral blood as CTCs is one of the main causes of cancer recurrence (2). Metastasis involves the migration of cancer cells away from primary tumors and their entry into blood circulation. Tumor metastasis is a fundamental cellular process for maintaining tissue homeostasis by removing displaced epithelial or endothelial cells, thus preventing them from seeding to inappropriate sites (3). In vivo and in vitro studies have demonstrated that the capability of cells to resist anoikis (death after detachment) is critical for successful metastasis (4-6).

Anoikis resistant cancer cells can often survive and grow without adhesion to the basement membrane as aggregated microemboli in the bloodstream and such growth represents a hallmark of the malignant phenotype (7). A previous study has demonstrated that anoikis resistance in CTCs was accompanied by spheroid formation and caspase-3/9/poly (ADP ribose) polymerase (PARP) inactivation (8). Furthermore, E-cadherin and $\beta$-catenin are important intracellular signaling molecules associated with cell aggregation (9), which itself is associated with colony formation as cell clusters and indicative of poor prognosis (10). Micrometastases resulting from such tumor cell aggregates by $\beta$-catenin/E-cadherin activation are thought to survive within the circulation as small cell clusters or spheroids, thereby effecting suppression of anoikis (11). It has suggested that the disruption of anoikis resistance may serve as a therapeutic strategy for the treatment of malignant cancer (12).

Monascin(MS) is a yellow pigment produced by Monascus; it exhibits diverse pharmacological activities, including anti-inflammatory activity and antioxidant activity (13), and induction of cell death in cancer cells (14). However, the effects of MS on CTCs have not been elucidated. In the present study, the ability of MS to induce anoikis was investigated in murine anoikis-resistant 4T1 breast cancer cells. The biochemical mechanisms underlying anoikis induction by MS were also determined. The results of the present study may lead to the development of novel strategies for the CTC metastasis treatment and prevention.

\section{Materials and methods}

Reagents. Monascin (cat. no. 52442) and other chemicals and reagents (unless otherwise stated) were purchased from Sigma-Aldrich; Merck KGaA. Media, FBS (cat. no. 26140079) and culture supplements were purchased from Invitrogen; 
Thermo Fisher Scientific, Inc. Anti-PARP (cat. no. 9542), caspase-3 (cat. no. 9665), E-cadherin (cat. no. 3195), $\beta$-catenin (cat. no. 9562) and $\beta$-actin (cat. no. 4970) were purchased from Cell Signaling Technology, Inc.

Cell culture. The murine breast cancer $4 \mathrm{~T} 1$ cell line was purchased from the American Type Culture Collection and the normal murine mammary gland (NMuMG) cell line was purchased from Bioresource Collection and Research Center. Each cell line was cultured in DMEM supplemented with $10 \% \mathrm{FBS}$ and $100 \mathrm{U} / \mathrm{ml}$ streptomycin/penicillin. Cells were incubated at $37^{\circ} \mathrm{C}$ in an atmosphere of $5 \% \mathrm{CO}_{2}$. For obtaining an anchorage-independent culture, cells were cultured in the same complete medium but on a poly [2-hydroxyethyl methacrylate (polyHEMA)]-coated plate. Briefly, polyHEMA was dissolved in $95 \%$ ethanol to obtain $12 \%$ (w/v) stock solution. Working solution was prepared by further dilution (1:10) of stock solution using 95\% ethanol and added to tissue culture dishes or plates ( $3 \mathrm{ml}$ per $10 \mathrm{~cm}$ dish or $0.5 \mathrm{ml}$ per well in a six-well plate). A hydrophobic surface was formed after polyHEMA solution was evaporated at room temperature in a tissue culture hood.

Cell proliferation assay. Both 4T1 and NMuMG cell proliferation rates were detected using the Cell Counting kit-8 (CCK-8) assay (Dojindo Molecular Technologies, Inc.). Cells were seeded into a $96-$ well plate at a density of $5 \times 10^{3}$ cells/well. After culturing for $24 \mathrm{~h}, 48 \mathrm{~h}$ and $72 \mathrm{~h}$ at $37^{\circ} \mathrm{C}, 10 \mu \mathrm{l}$ of CCK-8 solution was added to the completed medium and cells were further incubated at $37^{\circ} \mathrm{C}$ for $1 \mathrm{~h}$. Cell viability was determined by measuring absorbance at $450 \mathrm{~nm}$ on a microplate reader.

Trypan blue exclusion assay. 4T1 and NMuMG cells were cultured in each well $\left(1 \times 10^{6}\right.$ cells/well $)$ of a 6 -well plate with or without a polyHEMA coating. After $24 \mathrm{~h}$ incubation, culture medium was discarded and cells were washed with PBS and suspended in trypan blue stock in PBS (final concentration, $4 \%)$. Diluted trypan blue solution $(0.04 \%)$ was subsequently added to the 6 -well plate ( $500 \mu \mathrm{l} /$ well). After 3 min incubation at room temperature, stained cells were observed under a light microscope at a magnification of x10. Dead cells were dyed blue and viable cells were colorless and transparent.

Annexin V-FITC and PI double staining assay. After $48 \mathrm{~h}$ treatment with $20 \mu \mathrm{M}$ MS, 4T1 cells were harvested via trypsinization and washed with cold PBS. Cells were then centrifuged at $300 \mathrm{x} \mathrm{g}$ for $5 \mathrm{~min}$ at $4^{\circ} \mathrm{C}$, after which the supernatant was discarded and the pellet was resuspended in $1 \mathrm{X}$ binding buffer. Sample solution $(60 \mu \mathrm{l})$ was incubated with $3 \mu$ l FITC-conjugated Annexin V (BD Pharmingen; BD Biosciences) and $1 \mu \mathrm{g} / \mathrm{ml}$ PI (Sigma-Aldrich; Merck KGaA; cat. no. P4170) for $15 \mathrm{~min}$ at room temperature in the dark. A total of $240 \mu \mathrm{l} 1 \mathrm{X}$ binding buffer was added to each tube and the samples were counted (20,000 cells) and analyzed using a CytoFLEX ${ }^{\mathrm{TM}}$ Flow Cytometer and CytExpert software version 2.4 (Beckman Coulter, Inc.).

Western blot analysis. 4T1 cells were lysed in ice-cold radioimmunoprecipitation assay lysis buffer (Merck KGaA; cat. no. 632424) containing protease inhibitor cocktails (EMD Millipore; cat. no. 539134). Protein concentration was determined using Bicinchoninic Acid Protein Assay Kit (Santa Cruz Biotechnology, Inc.; cat. no. sc-202389). Proteins (20 $\mu \mathrm{g})$ were separated by $10 \%$ SDS-PAGE and transferred onto PVDF membranes. Membranes were washed with PBS supplemented with $0.1 \%$ Tween-20. After blocking with BlockPRO ${ }^{\mathrm{TM}}$ Protein-Free Blocking Buffer (Visual Protein; cat. no. BF01) for $1 \mathrm{~h}$ at room temperature, membranes were incubated with primary antibodies against PARP (1:1,000; Cell Signaling Technology, Inc.; cat. no. 9542), caspase-3 (1:1,000; Cell Signaling Technology, Inc.; cat. no. 9665), E-cadherin (1:1,000; Cell Signaling Technology, Inc.; cat. no. 3195), $\beta$-catenin (1:1,000; Cell Signaling Technology, Inc.; cat. no. 9562) and $\beta$-actin (1:2,000; Cell Signaling Technology, Inc.; cat. no. 4970) primary antibodies overnight at $4^{\circ} \mathrm{C}$. Membranes were then incubated with anti-rabbit IgG HRP-linked antibody (1:5,000; Cell Signaling Technology, Inc.; cat. no. 7074) for $1 \mathrm{~h}$ at room temperature. Immobilon Western Chemiluminescent HRP Substrate (EMD Millipore; cat. no. WBKLS0500) was used to detect the signal on the membrane that were developed on Hyperfilm $^{\mathrm{TM}}$ ECL $^{\mathrm{TM}}$ film (GE Healthcare; cat. no. 28906839).

Animal model. All animal care and experimental procedures adhered to the guidelines of the Institutional Animal Care and Use Committee of Wan Fang Hospital, Taipei Medical University (Taipei, Taiwan; approval no. WAN-LAC-106-012). Female 6-week-old BALB/cByJNar1 mice were purchased from the National Laboratory Animal Center (Taipei, Taiwan). Animals were given a standard laboratory diet and distilled water ad libitum under a 12-h light/dark cycle at $22 \pm 2^{\circ} \mathrm{C}$ and humidity $(55 \pm 5 \%)$ in the animal facility of Wan Fang Hospital. Stable 4T1-Luc cells were established as described previously (15). A total of 20 mice were used ( $\sim 18 \mathrm{~g}$ weight). The tail veins of mice were injected with $1 \times 10^{6} 4 \mathrm{~T} 1$-Luc cells on day 0 . The mice were then divided into four groups on day $1(\mathrm{n}=5$ per group): Vehicle control, tumor control, MS (100 mg/kg) and MS $(500 \mathrm{mg} / \mathrm{kg})$ groups. MS was fed to the mice via oral gavage once daily, 5 days per week for a total of 4 weeks.

Prior to in vivo bioluminescence imaging, mice were anesthetized with isoflurane in an acrylic chamber using a $4 \%$ isoflurane/air mixture for induction. Mice were injected intraperitoneally with substrate D-luciferin (Sigma-Aldrich; Merck $\mathrm{KGaA}$; cat. no. L9504) solution $(150 \mathrm{mg} / \mathrm{kg}$ ) in Dulbecco's Phosphate-Buffered Saline (Sigma-Aldrich; Merck KGaA; cat. no. D8537) and maintained under $2 \%$ isoflurane. After $5 \mathrm{~min}$, images of the live anesthetized mice were recorded using a bioluminescence IVIS Spectrum System (PerkinElmer, Inc.; part no. 124262), which included a cryogenic cooling unit and a data acquisition computer with Living Image software version 2.5 (Xenogen Corp.). The acquisition and overlay of pseudocolor images were conducted. Images represented the spatial distribution of detected photons emerging from active luciferase within the animals. Bioluminescent signals were quantified using Living Image software 2.5 (Xenogen Corporation) as photons/sec/region of interest on days 7, 14,21 and 28 of treatment.

Statistical analyses. All statistics were calculated using GraphPad Prism 6 software (GraphPad Software, Inc.). P $<0.05$ was considered to indicate a statistically significant difference. Data are presented as mean \pm SD of triplicate experiments. Statistical significance for parametric data were determined 
A
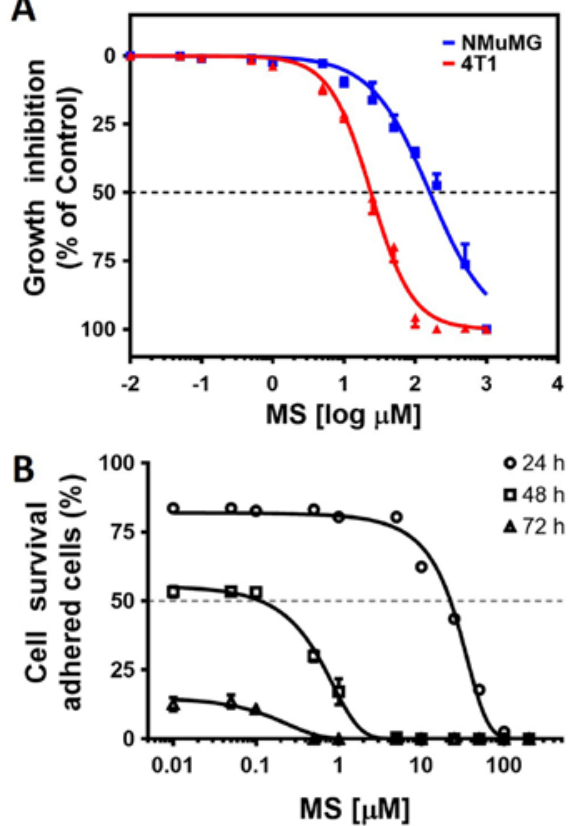

C

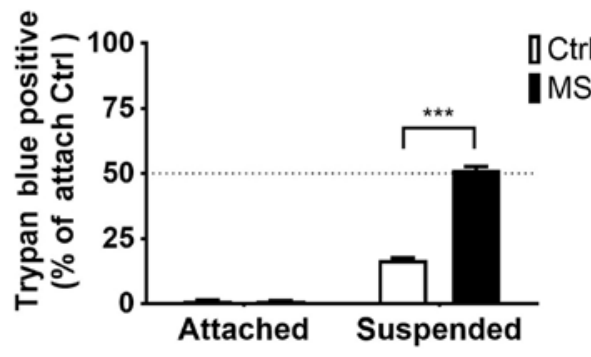

Figure 1. Inhibitory effects of MS on the viability of breast cells. (A) 4T1 breast cancer cells and NMuMG normal breast cells were treated with MS for $48 \mathrm{~h}$. A growth inhibition of $50 \%$ was observed at 23.8 and $160.0 \mu \mathrm{M}$ in $4 \mathrm{~T} 1$ and NMuMG cells, respectively. (B) Cell survival of $4 \mathrm{~T} 1$ cells cultured on the polyHEMA-coated plates treated with MS for 24, 48, and $72 \mathrm{~h}$ was compared with that of the cells under normal adhesion conditions at the same time points. (C) Trypan blue dye exclusion was used to measure the percentage of dead cells in different culture types treated with and without $20 \mu \mathrm{M}$ MS for $48 \mathrm{~h}$. The relative percentage of dead cells under the suspension condition was compared with that under the attachment condition. Points indicate the mean \pm standard deviation of at least three independent experiments that were each performed in triplicate. ${ }^{* * *} \mathrm{P}<0.001$ vs. control. MS, monascin; Ctrl, control.

using two-way ANOVA with Tukey's multiple comparisons test to compare multiple dependent variables against multiple independent variables. An unpaired two-tailed t-test was used to compare one dependent variable against one independent variable. Error bars represent the standard error of the mean obtained from experiments performed in triplicate.

\section{Results}

Anoikis-sensitization effect of MS in 4T1 breast cancer cells. The cytotoxic effect of MS was evaluated using murine NMuMG normal breast cells and 4T1 breast cancer cells treated with increasing concentrations $(0.1-1,000 \mu \mathrm{M})$ of MS for 48 h. MS markedly suppressed the survival of 4T1 breast cancer cells without causing toxic damage to normal NMuMG cells (Fig. 1A). To further validate that MS acted as an anoikis sensitizer, adherent and suspended breast cells were treated with increasing concentrations $(0.01-100 \mu \mathrm{M})$ of MS for 24-72 h. The number of dead cells in the MS-treated samples increased with the MS concentration (10-100 $\mu \mathrm{M})$; thus, MS exhibited cytotoxicity in a dose- and time-dependent manner (Fig. 1B). The results were further confirmed by using the trypan blue exclusion method to count the number of dead cells. 4T1 cells treated with $20 \mu \mathrm{M}$ MS for $24 \mathrm{~h}$ on polyHEMA-coated culture plates (suspended cells) were more sensitive to anoikis than attached cells (Fig. 1C).

MS induces caspase-dependent apoptosis in a suspension culture of highly metastatic breast cancer cells. 4T1 breast cancer cells were plated on uncoated (attached) or polyHEMA-coated (suspended) tissue culture plates for $48 \mathrm{~h}$. To confirm the occurrence of apoptosis, an
Annexin V-FITC/PI double staining assay was performed. The percentage of apoptotic cells (early and late apoptotic cells) in the polyHEMA-coated plate increased significantly from 9.91 to $44.96 \%$ after treatment with $20 \mu \mathrm{M}$ MS (Fig. 2A). As caspase activation is considered a hallmark of apoptosis (16), western blotting was performed to examine caspase activity. Cleaved caspase-3 expression clearly increased in the cells in the polyHEMA-coated plates after MS treatment. Cleaved PARP was also notably activated in 4T1 cells treated with $20 \mu \mathrm{M}$ MS under the suspension condition (Fig. 2B).

MS inhibits breast cancer cell aggregation under the suspension condition. In suspension culture, 4T1 cells formed large aggregates, but the cells did not exhibit aggregation after MS treatment (Fig. 3A). These observations suggest that cell aggregation provided growth signals to the tumor cells in suspension. To understand the molecular nature of the survival and growth signals provided by 4T1 cell aggregation, survival pathways were analyzed. The data indicated that MS reduced cell aggregation, which facilitated anoikis and downregulated the expression of E-cadherin and $\beta$-catenin (Fig. 3B).

MS treatment reduces lung metastasis in 4T1-Luc animal model. Considering that 4T1 aggregation was inhibited by MS treatment in vitro (Fig. 3A), the in vivo effect of $\mathrm{MS}$ on lung metastasis was determined. 4T1 cells were transfected with the firefly luciferase gene (Luc) in an experimental metastasis model. 4T1-Luc cells were injected into the tail vein of the mice. Non-invasive bioluminescence imaging enabled the early detection of cancer metastasis. Weekly bioluminescence imaging was conducted for 4 weeks, and the radiance antemortem was used as a surrogate measurement of tumor 

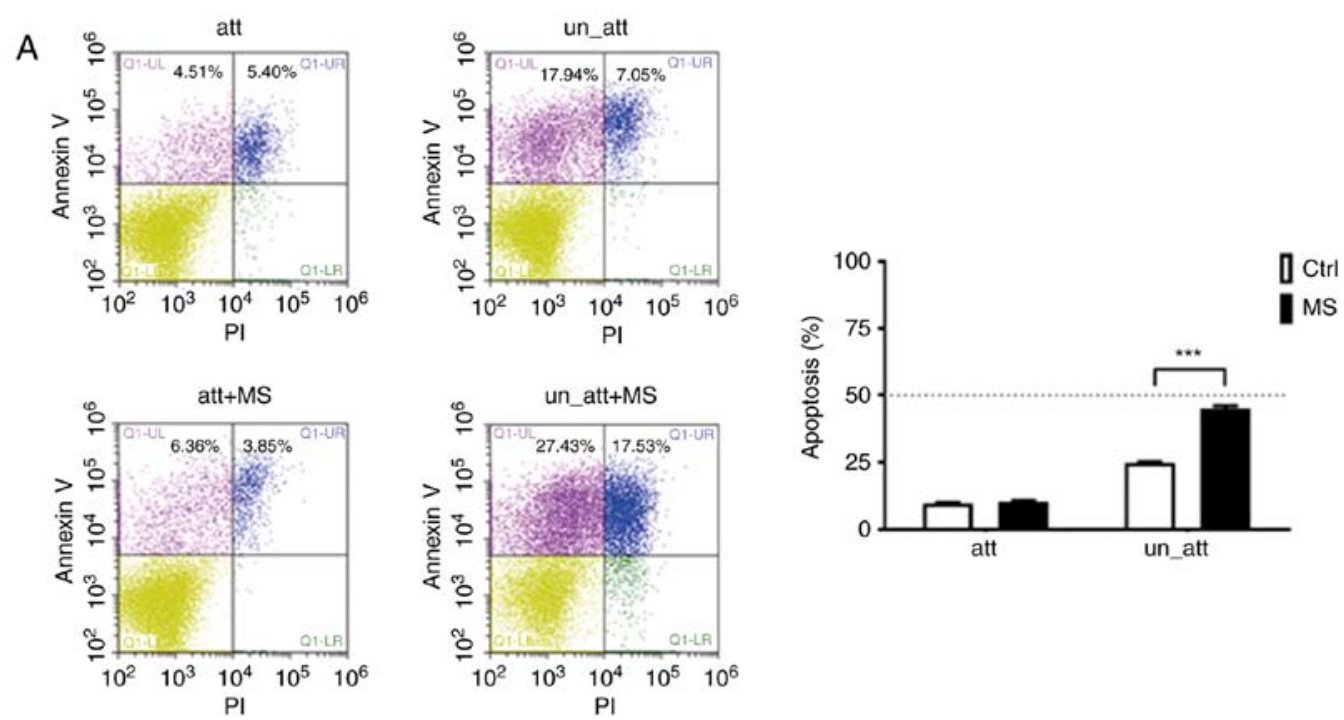

B Poly-HEMA

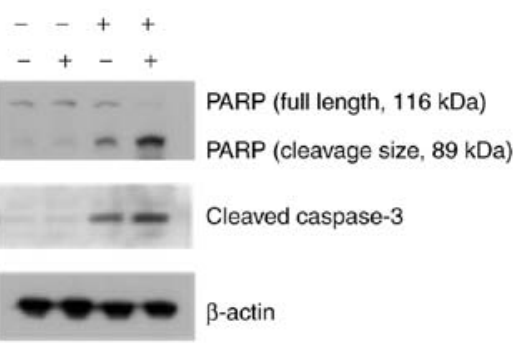

Figure 2. Induction of apoptosis by MS in $4 \mathrm{~T} 1$ breast cancer cells. (A) Fluorescence-activated cell sorting analysis of Annexin V/PI staining. The results indicated early apoptosis, defined as Annexin V-positive and PI-negative cells (Q1-UL), and late apoptosis, defined as Annexin V-positive and PI-positive cells (Q1-UR). 4T1 cells were cultured on a general culture plate (att) or a polyHEMA-coated plate (un_att), treated with $20 \mu \mathrm{M}$ (MS) or without MS for $48 \mathrm{~h}$, and then labeled with Annexin V-FITC and PI. The results are expressed as means \pm SD of three independent experiments. ${ }^{* * *} \mathrm{P}<0.001$ vs. control.(B) Western blot analysis for determining the effect of MS treatment for $48 \mathrm{~h}$ on PARP and cleavage caspase-3 expression under attachment and suspension conditions. MS, monascin; Ctrl, control; att, attached; un_att, unattached; PI, propidium iodide.

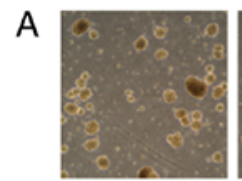

Ctrl

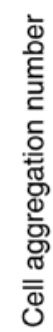

Figure 3. MS inhibits $4 \mathrm{~T} 1$ breast cancer cell aggregation under the suspension condition. (A) Phase-contrast images of $4 \mathrm{~T} 1$ cells on the polyHEMA-coated plate treated with or without $20 \mu \mathrm{M}$ MS for $48 \mathrm{~h}$. Quantitative counts of clustered $4 \mathrm{~T} 1$ cells reveal a significant decrease in MS treatment. (B) Western blot analysis of E-cadherin and $\beta$-catenin expression to determine the effect of MS treatment for $48 \mathrm{~h}$ on the floating cells. " $\mathrm{P}<0.05$ vs. control. MS, monascin; Ctrl, control.

burden. The mice treated with MS (100-500 mg/kg daily) exhibited a notable reduction in both the number and size of pulmonary metastases, compared with the vehicle-treated mice (Fig. 4).

\section{Discussion}

Anchorage independence is a well-known characteristic of CTCs that allows floating cancer cell metastasis (1). Based on this assumption, anoikis resistance may be a crucial early characteristic of malignant cancer, because cancer cells are either deprived of extracellular matrix or exposed to foreign matrix components during metastasis (17). Moreover, anoikis resistance is associated with a high degree of tumor metastasis and advanced stage of cancers (18). Therefore, targeting anoikis-resistance pathways represents a promising strategy for antimetastatic therapy. In previous report, we have descripted that the count of CTCs is a significant predictor for liver metastasis within six months of surgery (19). Due to the difficulty in establishing the primary tumor circulating cells, the 4T1 breast cancer cell line was used to develop CTC-like model. Similarly, in another study performed by Park et al (20), stable CTC-like cells derived from human breast cancer MDA-MB-468 cell line were established. Therefore, the effects of MS on 4T1 cells aggregation was examined via poly-HEMA coating culture. The present results indicated the suspended cells, forced to grow under anchorage-independent conditions, exhibited upregulated $\beta$-catenin/E-cadherin expression and downregulated expression of the intrinsic apoptosis pathway, via inhibiting PARP/caspase-3 cleaved as a mechanism for suppressing anoikis. To the best of our 
<smiles>C/C=C/C1=CC2=C(CO1)C(=O)[C@@]1(C)OC(=O)C(C(=O)CCCCC)[C@H]1C2</smiles>

Monascin (MS)

Pre-treatment for 1 week -
4T1-Luc cells i.v. injection and combination with treatment for 4 weeks

B

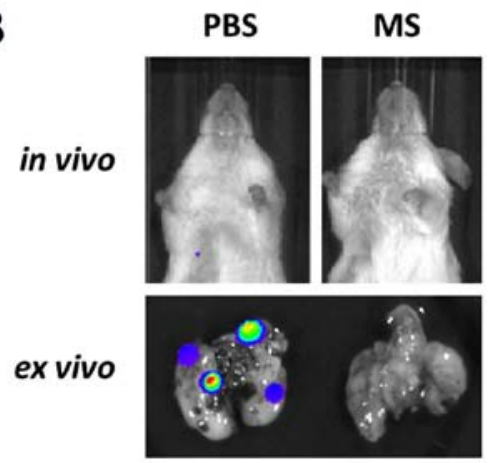

C D14 PBS es D21 D28

MS 100
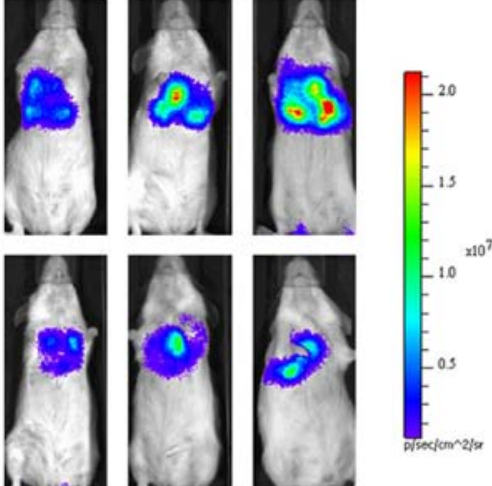

MS

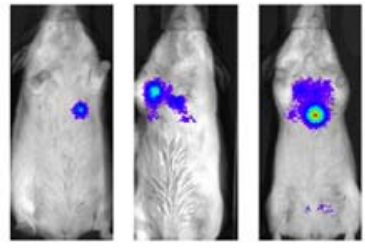

Figure 4. MS treatment reduces tumor growth in a tail vein-injected mouse model. (A) Before injection of 4T1 cells expressing the firefly luciferase gene (4T1-Luc), female BALB/c mice were treated (oral gavage) with 100 or $500 \mathrm{mg} / \mathrm{kg}$ MS (or vehicle PBS control) for 1 week. Then, the mice were intravenously injected with 4T1-Luc cells $\left(1 \times 10^{5}\right)$ through the tail vein, and the treatment (MS or vehicle PBS) continued for 4 weeks. (B) For 1 week after tumor injection, bioluminescence signals were not detected in any of the living mice. Pulmonary metastasis susceptibility was observed via detection of bioluminescence signals in the vehicle PBS-treated mice after sacrifice; however, no signal was detected in the MS-treated groups. (C) Bioluminescence signals [mean photon flux (photons/s)] were assessed in the areas over the lungs on days 14, 21 and $28 . \mathrm{n}=5$ mice per group. MS, monascin.

knowledge, this was the first report of MS resensitizing breast cancer cells to anoikis and reducing colony-forming ability under a suspension condition.

The secondary metabolite MS produced by Monascus species has been reported to exhibit multiple biological effects, including anti-inflammatory effects (21). In the present study, the effects of MS on tumor growth and metastasis in 4T1 metastatic tumor-bearing mice were investigated, and the effects on cell adhesion, anoikis resistance and cell migration in 4T1 breast cancer cells were examined. In the current study, it was revealed that MS restored anoikis sensitivity in suspended 4T1 cancer cells. Notably, MS inhibited the proliferation of 4T1 cells but was not cytotoxic to normal NMuMG cells. Also, MS selectively induced cell death in suspended 4T1 cells and MS-induced cell death was demonstrated to be due to apoptosis, as demonstrated by the high levels of proapoptotic caspase activation and PARP cleavage. Finally, MS treatment prevented the growth of floating 4T1-Luc cells injected into the tail vein of the experimental mice and inhibited the migration of the cells to the lungs.

However, there were a few limitations to the present study. The current results were not derived from isolated primary circulating tumor cells, and clinical data was not available to support the in vitro and in vivo findings. Further studies are required to investigate the effect of MS treatment on clinical breast cancer metastasis, and this should be confirmed in a clinical trial. Multiple animal models have been developed recently, but these models are not designed appropriately to study the step-by-step progression of metastasis (22). In this complicated metastasis process, the present study only describes the anoikis resistance on circulating tumor cells. Despite having certain limitations, such as the step of intravasation from primary site into the bloodstream, the poly-HEMA coating culture-induced cell cluster formation may be an in vitro experimental model for anoikis resistance investigation.

Previous studies on tumor metastasis and the epithelial-mesenchymal transition (EMT) have reported that EMT enables cancer cells to migrate away from the primary tumor (23-25). However, whether cell detachment additionally triggers EMT remains unclear. In the present study, high levels of $\beta$-catenin expression following detachment may be associated with cell aggregation and proliferation. $\beta$-Catenin is a versatile protein that serves multiple fundamental functions in cells, such as controlling intercellular junction integrity and regulating transcriptional processes as a co-transcription factor, mediating the canonical Wnt signaling pathway (26). Thus, targeting $\beta$-catenin activity and reducing anoikis resistance may represent strategies for preventing the development and progression of breast cancer. A previous study has revealed that disruption of E-cadherin-mediated adhesion sensitizes multicellular spheroids of tumor cells to treatment with chemotherapeutic drugs (27). MS, with the function to downregulate E-cadherin, may therefore have potential as a neoadjuvant for clinical metastatic prevention after surgery. 
In conclusion, the current results demonstrate that MS targeting anti-anoikis may increase the killing of breast tumor cells persisting in the circulation as anchorage-independent micrometastasis. Future studies are needed on MS, including evaluation of anoikis resistance as a clinical application.

\section{Acknowledgements}

Not applicable.

\section{Funding}

The present study was supported by grants from Wan Fang Hospital, Taipei Medical University (grant nos. 106-swf-05, 106-swf-11 and 108-wf-eva-09) and the Ministry of Science and Technology, Taiwan (grant no. MOST 109-2314-B-038-039).

\section{Availability of data and materials}

The datasets used and analyzed during the current study are available from the corresponding author on reasonable request.

\section{Authors' contributions}

KYC, YTT and BYH conceived and designed the study. KYC, JAL and BYH performed animal experiments. HYY and $\mathrm{ACH}$ assisted in the animal experiments. YTT and BYH directed the project, analyzed and interpreted the data and wrote the manuscript. All authors read and approved the final manuscript.

\section{Ethics approval and consent to participate}

All animal care and experimental procedures adhered to the guidelines of the Institutional Animal Care and Use Committee of Wan Fang Hospital, Taipei Medical University (approval no. WAN-LAC-106-012, Taipei, Taiwan).

\section{Patient consent for publication}

Not applicable.

\section{Competing interests}

The authors declare that they have no competing interests.

\section{References}

1. Aceto N, Bardia A, Miyamoto DT, Donaldson MC, Wittner BS, Spencer JA, Yu M, Pely A, Engstrom A, Zhu H, et al: Circulating tumor cell clusters are oligoclonal precursors of breast cancer metastasis. Cell 158: 1110-1122, 2014.

2. Cristofanilli M, Budd GT, Ellis MJ, Stopeck A, Matera J, Miller MC, Reuben JM, Doyle GV, Allard WJ, Terstappen LW and Hayes DF: Circulating tumor cells, disease progression, and survival in metastatic breast cancer. N Engl J Med 351: 781-791, 2004.

3. van Dalum G, Holland L and Terstappen LW: Metastasis and circulating tumor cells. EJIFCC 23: 87-97, 2012.

4. Sirimangkalakitti N, Chamni S, Suwanborirux K and Chanvorachote P: Renieramycin M Sensitizes Anoikis-resistan H460 lung cancer cells to anoikis. Anticancer Res 36: 1665-1671, 2016.

5. Zhang X, Yang L, Chien S and Lv Y: Suspension state promotes metastasis of breast cancer cells by up-regulating cyclooxygenase-2. Theranostics 8: 3722-3736, 2018.
6. Zou G, Ren B, Liu Y, Fu Y, Chen P, Li X, Luo S, He J, Gao G, Zeng $Z$, et al: Inhibin B suppresses anoikis resistance and migration through the transforming growth factor- $\beta$ signaling pathway in nasopharyngeal carcinoma. Cancer Sci 109: 3416-3427, 2018.

7. Cominetti MR, Altei WF and Selistre-de-Araujo HS: Metastasis inhibition in breast cancer by targeting cancer cell extravasation. Breast Cancer (Dove Med Press) 11: 165-178, 2019.

8. Laguinge LM, Samara RN, Wang W, El-Deiry WS, Corner G, Augenlicht L, Mishra L and Jessup JM: DR5 receptor mediates anoikis in human colorectal carcinoma cell lines. Cancer Res 68: 909-917, 2008.

9. Steadman K, Stein WD, Litman T, Yang SX, Abu-Asab M, Dutcher SK and Bates S: PolyHEMA spheroids are an inadequate model for the drug resistance of the intractable solid tumors. Cell Cycle 7: 818-829, 2008

10. Hong Y, Fang F and Zhang Q: Circulating tumor cell clusters: What we know and what we expect (Review). Int J Oncol 49: 2206-2216, 2016.

11. Kang HG, Jenabi JM, Zhang J, Keshelava N, Shimada H, May WA, Ng T, Reynolds CP, Triche TJ and Sorensen PH: E-cadherin cell-cell adhesion in ewing tumor cells mediates suppression of anoikis through activation of the ErbB4 tyrosine kinase. Cancer Res 67: 3094-3105, 2007.

12. Giannoni E, Fiaschi T, Ramponi G and Chiarugi P: Redox regulation of anoikis resistance of metastatic prostate cancer cells: Key role for Src and EGFR-mediated pro-survival signals. Oncogene 28: 2074-2086, 2009.

13. Lee BH, Hsu WH, Huang T, Chang YY, Hsu YW and Pan TM: Effects of monascin on anti-inflammation mediated by $\mathrm{Nrf} 2$ activation in advanced glycation end product-treated THP-1 monocytes and methylglyoxal-treated wistar rats. J Agric Food Chem 61: 1288-1298, 2013.

14. SuNW,Lin YL,LeeMHandHoCY:AnkaflavinfromMonascus-fermented red rice exhibits selective cytotoxic effect and induces cell death on Hep G2 cells. J Agric Food Chem 53: 1949-1954, 2005.

15. Ho BY, Lin CH, Apaya MK, Chao WW and Shyur LF: Silibinin and paclitaxel cotreatment significantly suppress the activity and lung metastasis of triple negative 4T1 mammary tumor cell in mice. J Tradit Complement Med 2: 301-311, 2012.

16. Mohamed MS, Bishr MK, Almutairi FM and Ali AG: Inhibitors of apoptosis: Clinical implications in cancer. Apoptosis 22: 1487-1509, 2017

17. Kim YN, Koo KH, Sung JY, Yun UJ and Kim H: Anoikis resistance: An essential prerequisite for tumor metastasis. Int J Cell Biol 2012: 306879, 2012.

18. Piyush T, Rhodes JM and Yu LG: MUC1 O-glycosylation contributes to anoikis resistance in epithelial cancer cells. Cell Death Discov 3: 17044, 2017.

19. Tien YW, Kuo HC, Ho BI, Chang MC, Chang YT, Cheng MF, Chen HL, Liang TY, Wang CF, Huang CY, et al: A high circulating tumor cell count in portal vein predicts liver metastasis from periampullary or pancreatic cancer: A high portal venous CTC count predicts liver metastases. Medicine (Baltimore) 95: e3407, 2016.

20. Park JY, Jeong AL, Joo HJ, Han S, Kim SH,Kim HY,Lim JS, Lee MS, Choi HK and Yang Y: Development of suspension cell culture model to mimic circulating tumor cells. Oncotarget 9: 622-640, 2018.

21. Cheng CF and Pan TM: Monascus-fermented red mold dioscorea protects mice against alcohol-induced liver injury, whereas its metabolites ankaflavin and monascin regulate ethanol-induced peroxisome proliferator-activated receptor-gamma and sterol regulatory element-binding transcription factor-1 expression in HepG2 cells. J Sci Food Agric 98: 1889-1898, 2018.

22. Khanna $\mathrm{C}$ and Hunter $\mathrm{K}$ : Modeling metastasis in vivo. Carcinogenesis 26: 513-523, 2005.

23. Bill R and Christofori G: The relevance of EMT in breast cancer metastasis: Correlation or causality? FEBS Lett 589: 1577-1587, 2015.

24. Alix-Panabieres C, Mader S and Pantel K: Epithelial-mesenchymal plasticity in circulating tumor cells.J Mol Med (Berl) 95: 133-142,2017.

25. Melzer C, von der Ohe J and Hass R: Breast carcinoma: From initial tumor cell detachment to settlement at secondary sites. Biomed Res Int 2017: 8534371, 2017.

26. Gao C, Xiao $\mathrm{G}$ and $\mathrm{Hu} \mathrm{J}$ : Regulation of Wnt/ $\beta$-catenin signaling by posttranslational modifications. Cell Biosci 4: 13, 2014.

27. Green SK, Francia G, Isidoro $C$ and Kerbel RS: Antiadhesive antibodies targeting E-cadherin sensitize multicellular tumor spheroids to chemotherapy in vitro. Mol Cancer Ther 3: 149-159, 2004.

This work is licensed under a Creative Commons Attribution-NonCommercial-NoDerivatives 4.0 International (CC BY-NC-ND 4.0) License. 